\title{
Diabetes mellitus type II as a risk factor for depression: a lower than expected risk in a general practice setting
}

\author{
S. Aarts - M. van den Akker - M. P. J. van Boxtel • \\ J. Jolles $\cdot$ B. Winkens $\cdot$ J. F. M. Metsemakers
}

Received: 4 November 2008/Accepted: 18 August 2009/Published online: 29 August 2009

(C) The Author(s) 2009. This article is published with open access at Springerlink.com

\begin{abstract}
The aim of the present study was to determine whether a diagnosis of diabetes mellitus (DM) in a primary setting is associated with an increased risk of subsequent depression. A retrospective cohort design was used based on the Registration Network Family Practice (RNH) database. Patients diagnosed with diabetes mellitus at or after the age of 40 and who were diagnosed between 01-01-1980 and 01-01-2007 $(N=6,140)$, were compared with agematched controls from a reference group $(N=18,416)$ without a history of diabetes. Both groups were followed for an emerging first diagnosis of depression (and/or depressive feelings) until January 1, 2008. 2.0\% of the people diagnosed with diabetes mellitus developed a
\end{abstract}

S. Aarts $(\bowtie) \cdot$ M. van den Akker · J. F. M. Metsemakers Department of General Practice, School for Public Health and Primary Care: Caphri, Maastricht University,

P.O. Box 616, 6200 MD Maastricht, The Netherlands

e-mail: Sil.Aarts@HAG.unimaas.nl

M. van den Akker

e-mail: Marjan.vandenakker@HAG.unimaas.nl

J. F. M. Metsemakers

e-mail: Job.Metsemakers@HAG.unimaas.nl

M. P. J. van Boxtel · J. Jolles

Department of Psychiatry and Neuropsychology, School for Mental Health and Neuroscience, Maastricht University, Maastricht, The Netherlands

e-mail: M.vanboxtel@np.unimaas.nl

J. Jolles

e-mail: j.jolles@psy.vu.nl

\section{B. Winkens}

Department of Methodology and Statistics, School for public Health and Primary Care: Caphri, Maastricht University,

Maastricht, The Netherlands

e-mail: bjorn.winkens@stat.unimaas.nl depressive disorder, compared to $1.6 \%$ of the reference group. After statistical correction for confounding factors diabetes mellitus was associated with an increased risk of developing subsequent depression (HR 1.26; 95\% CI: 1.12-1.42) and/or depressive feelings (HR 1.33; 95\% CI: 1.18-1.46). After statistical adjustment practice identification code, age and depression preceding diabetes, were significantly related to a diagnosis of depression. Patients with diabetes mellitus are more likely to develop subsequent depression than persons without a history of diabetes. Results from this large longitudinal study based on a general practice population indicate that this association is weaker than previously found in cross-sectional research using self-report surveys. Several explanations for this dissimilarity are discussed.

Keywords Depressive disorder - Depressive feelings · Diabetes mellitus - General practice

\section{Abbreviations \\ DM Diabetes mellitus \\ GP General practitioner}

It is generally known that co-morbid depression is highly prevalent in persons diagnosed with a chronic illness [1]. Especially, the co-occurrence of depression in persons diagnosed with diabetes mellitus has been a major topic in recent epidemiologic research. This research suggests that depression is more prevalent among adults with diabetes than in those without this condition [2-4]. More precisely, a review and meta-analysis involving 42 cross-sectional studies by Anderson et al. [1] suggests that diabetes doubles the odds of a co-morbid depression [5]. 
The impact of depression on patients with diabetes has also been an area of interest. Gonzales et al. [11] defined co-morbid depression as a risk factor for nonadherence to important aspects of diabetes self-care, such as medication and diet regimens [6]. In addition, diabetic patients with a depression reported a greater number of physical symptoms related to diabetes [4], and showed lower levels of metabolic control [7] and quality of life [8].

In the past decade, literature on the association between diabetes mellitus and depression has grown considerably. However, the temporal or causal relationship between diabetes mellitus and depression still remains unclear since only few longitudinal studies have evaluated diabetes as a risk factor for depression [9]. Most studies that were of longitudinal nature focused on the relation between depression and the development of a subsequent diagnosis of diabetes mellitus [10-12] and have produced contradictory results $[10,13,14]$. The few studies that did focus on the longitudinal relation between diabetes and subsequent depression, showed that diabetes type II is associated with an increased risk of developing depressive symptoms $[13,15,16]$. However, these studies were either restricted to small populations [16] or lacked a proper adjustment for the presence of other chronic diseases [13, 15]. Consequently, the results of these studies must be considered less representative for the general population.

Finally, another major drawback in previous mentioned longitudinal studies concerns the use of self-report surveys to identify depression (e.g. CES-D [11, 13, 16], HADS [6], BDI-II [17], PHQ [4]) or self-reported diabetes [16]. An important consequence of this differential assessment is the wide variability in the presence and strength of the established association between diabetes and depression [11]. Besides, self-report surveys may not be the most appropriate measure for the assessment of depressive disorder for several reasons. Data from selfreport scales cannot be used for making a clinical diagnosis of depression. Moreover, by using different cut-off points to define depression [5], comparison between these studies is hindered. Finally, health questionnaires are known to suffer from several conceptual problems. For example, the results of self-reports and questionnaires are based on a particular point in time that frequently do not include information about the intensity or consistency of the complaints or problems, personal growth or coping strategies [18].

In the present study, the previously mentioned drawbacks were defied. The aim of the present study was to assess the risk of depression over time in patients with diabetes mellitus compared with those without a history of diabetes. For this purpose, a large retrospective cohort study was performed in a large general practice setting.

\section{Methods}

Sample frame

The present study was carried out within the context of the Registration Network Family Practices (RegistratieNet Huisartspraktijken, RNH). Descriptive background characteristics of samples in other studies which made use of the RNH database were found to be comparable to the Dutch population [19]. The RNH is a continuously updated database, which contains the medical records of patients from 21 family practices in which 65 general practitioners (GPs) working in the south of the Netherlands are participating. This database includes all relevant current and past health problems. A health problem is defined as 'anything that has required, does or may require health care management and has affected or could significantly affect a person's physical or emotional well-being' [20]. These problems are coded in a standardized fashion, according to the International Classification of Primary Care (ICPC), using the criteria of the International Classification of health in Primary Care (ICHPPC-2) [21] and other more current guidelines of the Dutch College of GP's. A diagnosis is made by the GP or by a medical specialist who is consulted by the GP. Especially in complex medical conditions, registrations is often based on a specialist diagnosis reported to the GP. In general, health problems are only coded by the GP when they are permanent (no recovery expected), chronic (duration longer than 6 months) or recurrent (more than three recurrences within 6 months), or when they have lasting consequences for the functional status or prognosis of the patient. The database also contains background information on the patient's sex, date of birth, marital status, type of household, practice identification code and level of education. Membership of the RNH population ends by migration or death. All patients included in the RNH database have been informed about the anonymous use of their health information and are removed from the database if desired. The quality of the data is ascertained by ample instruction and training sessions, regular regional consensus groups, quality control audits, an online thesaurus available during data-entry and systematic control for erroneous or missing entries [20].

\section{Diagnostic criteria}

ICPC is now widely used in Europe as a diagnostic classification system, which has relations both with ICD-9 and with other ICD-9 derived systems being used in primary care [21]. Modified international criteria are followed, as expressed in the guidelines of the Dutch College of General Practitioners, for the diagnosis of Diabetes Mellitus (ICPC code T90) [21]. 
The diagnosis of DM requires an elevated glucose level [fasting plasma glucose levels of $124 \mathrm{mg} / \mathrm{dl}(6.9 \mathrm{mmol} / \mathrm{l})$ or more, a fasting capillary glucose level of $108 \mathrm{mg} / \mathrm{dl}$ $(6.0 \mathrm{mmol} / \mathrm{l})$ or more, or non-fasting plasma or capillary glucose level of $198 \mathrm{mg} / \mathrm{dl}(11.0 \mathrm{mmol} / \mathrm{l})]$ that is confirmed using a fasting glucose level a few days later. Since the disease contents and progress between type I and type II diabetes may vary considerably, the present study was aimed at patients with type II diabetes. In order to diminish the number of patients with DM type I in the present study, only patients diagnosed with DM at or after the age of 40 years were included.

The diagnosis of depressive disorder (ICPC code P76) was made in a diagnostic interview conducted by either a general practitioner or a specialist. According to ICPC criteria, patients should not be psychotic and comply with at least three of the following six criteria: (1) sadness or melancholy more than can be explained by the psychosocial stress, (2) suicidal thoughts or attempt, (3) indecisiveness, decreased interest in usual activities or diminished ability to think, (4) feelings of worthlessness, self-reproach, or inappropriate or excessive guilt, (5) early morning wakening, hypersomnia, or early morning fatigue, or (6) anxiety, hyperirritability, or agitation. The nine symptoms of a depressive episode described in the DSMIV of the American Psychiatric Association are equivalent to the six criteria of the RNH following ICPC code P76 [21]. Patients who presented themselves with chronic or recurrent depressive feelings, but who did not fully complied with the requirements for depressive disorder (ICPC code P76) were coded as having depressive feelings (ICPC code P03).

\section{Study design}

Data were drawn from the RNH database available on January 1, 2008. Patients with DM in our study were diagnosed with ICPC code T90 (Diabetes Mellitus Type I and Type II) between January 1, 1980 and January 1, 2007. In order to restrict the number of patients with type I diabetes, only patients with diabetes mellitus who were 40 years or older at the time of diagnosis were included $(N=6,140)[3,22]$.

The reference group consisted of subjects with no history of DM. In order to increase the power of the current study, individuals in the reference group were matched to the diabetes patients by age (year of birth) and were assigned the date of diagnosis of their matched counterparts as the starting date for their follow-up period.

Each patient was matched (according to year of birth) to 3 controls in the reference group $(N=18,416)$, except for 4 patients, who could only be matched to 2 controls from the reference group. Each subject was followed for an emerging first diagnosis of a depressive disorder. Followup ended on January 1, 2008, or earlier in case of a diagnosis of depressive disorder or due to censoring (i.e. migration or death).

In the sensitivity analyses, each subject was followed for an emerging first diagnosis of a depressive disorder or the emergence of depressive feelings (ICPC code P03). Follow-up ended on January 1, 2008, or earlier in case of a diagnosis of depressive disorder, depressive feelings or due to censoring (i.e. migration or death).

\section{Statistical analysis}

Statistical analyses were conducted using the SPSS statistical software package version 16.0 for Windows (SPSS Inc., Chicago, IL, USA). An independent $T$-test was used to examine a possible difference in mean follow-up time between the diabetes and the reference group.

Cox proportional hazards survival analysis was applied in which the effect of diabetes on depression was corrected for age (ranging from 40 to 97 years), gender, level of education (3 levels: low, intermediate, and high), number of chronic co-morbid diseases in 7 categories (ranging from 0 to 6 diseases, in which the last category coded for 6 or more diseases) (see Appendix Table 2), the practice where a patient was registered (practice identification code) and a diagnosis of depression preceding the start of followup. The latter variable coded for all subjects who had a diagnosis of depression (and/or depressive feelings in case of the sensitivity analysis) before the starting date of the follow-up period.

All potential confounders in the analyses were declared as categorical and coded into dummy variables. Since patients were matched with controls by age (in years), age was also declared as a categorical variable. Hazards ratios (HR) and 95\% confidence intervals (CI) are reported. For the potential confounders the $P$-value of the survival analysis was reported. Potential confounders who were not significantly related $(P$-values $>0.05)$ to a subsequent diagnosis of depression were excluded from the final analyses using a backward method.

Two kinds of analyses were conducted. The first analysis made use of the diagnosis of depression as event outcome. The second analysis was a sensitivity analysis, in which the diagnosis of depressive feelings, not complying with the requirements for depressive disorder, was pooled with the diagnosis of depression as event outcome.

\section{Results}

Descriptive characteristics of the study sample are reported in Table 1. A significant difference in time until diagnosis 
Table 1 Descriptive characteristics of study population
${ }^{a}$ Mean age at date of inclusion cohort

${ }^{b}$ For $30.6 \%$ of the diabetic group and $18.1 \%$ of the reference group this information was missing or not updated; low is defined as primary school and/or lower vocational education, medium as secondary school and/or medium level vocational education, high as higher vocational education and/or university

\begin{tabular}{lcc}
\hline Characteristics & $\begin{array}{c}\text { Patients } \\
(n=6,140)\end{array}$ & $\begin{array}{c}\text { Controls } \\
(n=18,416)\end{array}$ \\
\hline No. of subjects with depression preceding diabetes $(\%)$ & $325(5.3)$ & $863(4.7)$ \\
No. of subjects with depression after diabetes $(\%)$ & $122(2.0)$ & $295(1.6)$ \\
Mean age in years (SD) $^{\mathrm{a}}$ & $63.8(11.2)$ & $63.8(11.2)$ \\
Mean number of follow-up years (range) & $7.7(0-28)$ & $7.9(0-28)$ \\
Gender & & $8,519(46.3)$ \\
$\quad$ Males $(\%)$ & $2,953(48.1)$ & $9,897(53.7)$ \\
Females $(\%)$ & $3,187(51.9)$ & $3,325(18.1)$ \\
Number of co-morbid diseases $(\%)$ & & $3,844(20.9)$ \\
0 & $0(0.0)$ & $3,367(18.3)$ \\
1 & $0(0.0)$ & $2,580(14.0)$ \\
2 & $566(9.2)$ & $1,950(10.6)$ \\
3 & $1,003(16.3)$ & $1,377(7.5)$ \\
4 & $1,040(16.9)$ & $1,973(10.7)$ \\
5 & $929(15.1)$ &
\end{tabular}

of depressive disorder between the two groups was found $(P<0.001)$. The mean follow-up was 7.7 years $(\mathrm{SD}=$ $5.7)$ for the diabetes group and 7.9 years $(\mathrm{SD}=6.6)$ for the reference group. During the follow-up period, 122 patients $(2.0 \%)$ with diabetes mellitus and 295 persons (1.6\%) of the reference group developed a depressive disorder.

Gender, co-morbidity and level of education did not have a significant effect and were therefore removed as covariates from all statistical analyses.

A diagnosis of diabetes mellitus was significantly associated with a greater likelihood of developing subsequent diagnosis of depression (HR 1.32; 95\% CI: $1.19-$ 1.48). Statistical adjustment for the remaining confounders (age, practice identification code and depression preceding DM) attenuated the strength of this association, but it remained statistical significant (HR 1.26; 95\% CI: $1.12-$ 1.42) (see Fig. 1).

Adding depressive feelings to the event outcome yielded similar results. During follow up diabetes patients were significantly more likely to develop a depressive disorder and/or depressive feelings (HR 1.27; 95\% CI: 1.15-1.41). After controlling for confounding factors (age, practice identification code and depression preceding DM) this association became even slightly stronger (HR 1.31; 95\% CI: 1.18-1.46) (see Fig. 2). After adjusting for the other variables, practice identification $(P=0.004)$, age $(P<$ 0.001 ) and a diagnosis of depression preceding DM
$(P<0.001)$ code also showed a significant association with risk of depression.

\section{Discussion}

The present study revealed that a diagnosis of diabetes mellitus type II at or after the age of forty was associated with an increased likelihood of developing a subsequent depression or depressive feelings. After statistical adjustment, practice identification code, age and a diagnosis of depression preceding the diagnosis of diabetes mellitus appeared to be significantly related to a subsequent diagnosis of depression.

This is the first longitudinal study on the association between diabetes mellitus and subsequent depression based on data from general practices. A meta-analysis by Anderson et al. [1] involving 42 cross-sectional studies reported that patients with type II diabetes are twice more likely to experience depressive symptoms than their peers without diabetes. In contrast, the present results suggest that patients with diabetes mellitus are 1.12-1.41 (CI) times more likely to develop depression and 1.18-1.46 times (CI) more likely to develop depression and/or depressive feelings. Our findings confirm the outcome of earlier studies in showing an increased incidence of depression among patients diagnosed with diabetes mellitus. However, this association appears to be weaker than 


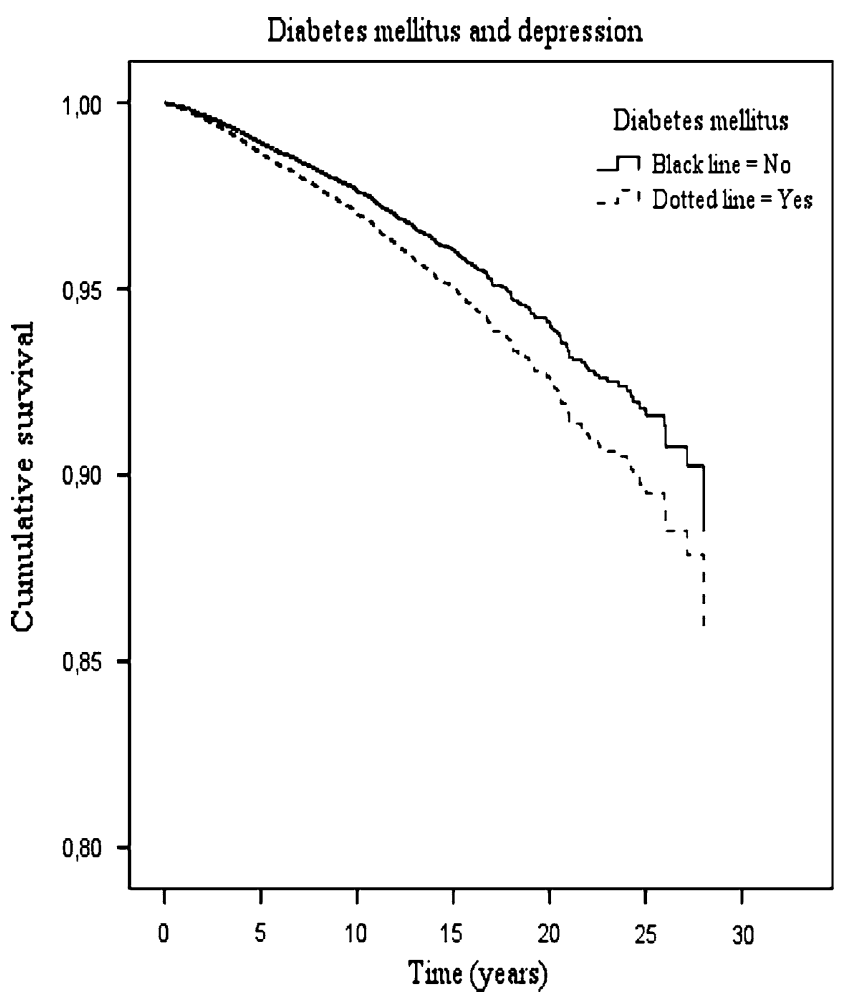

Fig. 1 Survival curve corrected for age, practice identification code and a diagnosis of depression preceding DM

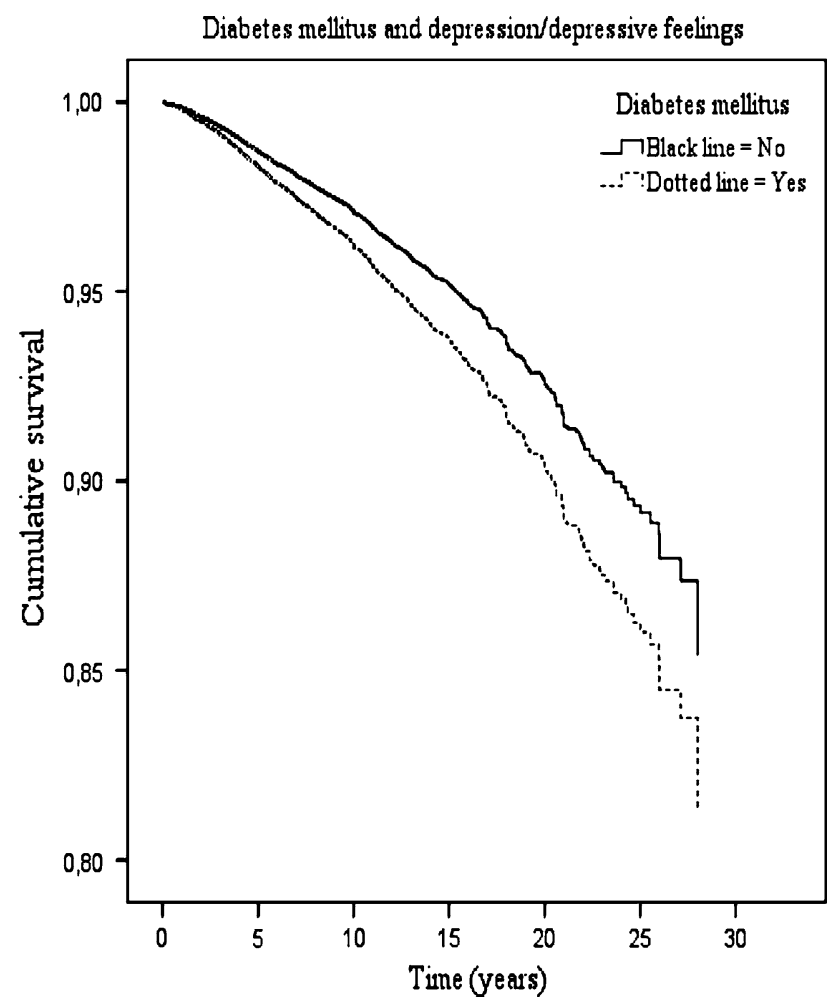

Fig. 2 Survival curve corrected for age, practice identification code and a diagnosis of depression preceding DM found in earlier studies. This discrepancy can be due to a number of reasons. Firstly, it has been shown that depression rates are two to three times higher in studies that use self-reports [5]. For example, self-report measures may identify a broader spectrum of depressive disorders or symptoms that may reflect co-morbid psychiatric illness or general distress [5], which could result in an overestimation of the prevalence of depression [13]. Secondly, using a diagnosis of depression preceding DM as a confounder can have reduced the association between diabetes type II and depression. Although the association between diabetes and depression attenuated without this correction, it remained statistical significant. Finally, in the present study GPs were not instructed to systematically screen patients for possible depression or depressive symptoms which could have led to a lower risk estimate. If this is indeed the case, the present study reports an underestimation of depression in diabetes patients. It is important for GPs to be aware of the fact that patients with type II diabetes are more likely to experience depression than their peers without diabetes. Several studies have shown that adherence to a variety of self-care activities [6] and metabolic control [7] decreases in diabetes patient as a consequence of co-morbid depression. Diabetes patients with co-morbid depression also show a decline in quality of life [8]. Moreover, for approximately $75 \%$ of the people diagnosed with diabetes mellitus in the Netherlands, the GP is the primary medical caregiver [23], making the GP the proficient person to detect depression or depressive feelings. The present findings imply that GPs who are consulted by patients diagnosed with DM type II should be especially aware of the patient's increased risk of developing depression in the near future. In short, general practitioners should be alert to possible early signs of depression in diabetic patients to ensure early detection and possibly even prevention of a depressive disorder. Nurse practitioners for diabetes mellitus, who assist the general practitioners and provide a broad range of health care services, could fulfill an important role in this aspect. Nurse practitioners focus on patients' conditions as well as on the effects of the illness on the lives of the patients and their families and can therefore serve as a "point of entry" for physical as well as mental problems diabetes patients encounter. Nurse practitioners could enhance diagnostics by systematically screen diabetes patients for possible depression or depressive symptoms in order to prevent under diagnosis of depression in diabetes patients.

Another intriguing finding of the present study was that practice identification code was significantly associated with an enhanced likelihood of developing a depression. It appears that in 3 of the 21 practices involved, a relatively high percentage of patients is diagnosed with depression (ranging from 9.5 to $9.8 \%$ of the patients) while in 3 of the 
21 practices this percentage is noticeably smaller (ranging from 4.0 to $4.7 \%$ of the patients). Hence, it seems plausible that GPs may differ regarding their inclination to diagnose a depression. After investigating the most important characteristics of the practices incorporated in the present study, such as geographic place (defined by postal code) of a general practice, total number of diagnosed depressive disorders in the general practice, number and gender of patients, education of the patients, and number and gender of GPs in a practice, we were not able to identify any specific characteristics that could explain this effect. This diagnostic variability may have important implications for general practices [24].

Our study has several advantages over previous studies. This is the first longitudinal study that evaluated the causal relationship between diabetes mellitus type II and subsequent occurrence of depressive disorder and/or depressive feelings in a general practice based setting. Consequently, the results seem more representative for the general population than results of studies conducted in smaller and more homogeneous samples [17, 25]. Also, the sample size used in the present study supports the robustness of our risk estimates. Moreover, prior studies commonly relied on a variety of self-report surveys which are known to overestimate the prevalence of depression [5]. The six criteria following diagnosis of depressive disorder in the RNH database are essentially comparable to the nine symptoms of a depressive episode described in the DSMIV of the American Psychiatric Association [21]. These criteria are a solid foundation for making a uniform diagnosis of depression by GPs.

Despite the previous mentioned strengths, our findings must be interpreted in light of some possible limitations. First, since the RNH database does not make a clear distinction between diabetes type I and type II, the present study only included patients diagnosed with diabetes mellitus at or after the age of 40 years. Consequently, it can not be ruled out that none of the diabetes type I patients were included. Second, the total number of conditions registered in the RNH database reflects the GPs perspective of the health status and relevant health problems of his patients. As a result, some health problems may be missing because the patient did not report them to the GP or because the GP does not judge them to be clinically significant [26]. The number of missing health problems, however, appears to be rather small [19]. Furthermore, GPs have a tendency to use a diagnosis primarily as a mean to reach the goal of helping the patient and not as a goal in itself [18]. This is not the case in questionnaires and self-reports, which could have resulted in an underestimation of the prevalence of depression. Due to the very large follow-up period (01-011980 to 01-01-2008) it may be argued that no state of the art impression of the association between diabetes and subsequent depression is given. However, an additional analysis involving a smaller, more recent period of time (01-01-1995 to 01-01-2008) yielded similar results. Moreover, an analysis with a follow-up period of only 6 months was also conducted, which gave similar results. Finally, one of the drawbacks of studies that combine data from multiple practices is the between practice variability [19]: it is generally assumed that some variability exists between general practitioners in making a diagnose [24]. Possibly, this between practice variability may have resulted in the effect that code of practice has on the development of depression.

Further research is warranted to investigate a multitude of unanswered questions. More well-conducted research with adequate control for confounding factors is needed to investigate the causal relationship between diabetes and depression mellitus more in depth. In particular, the influence of practice setting on the longitudinal association between diabetes and depression should be studied in more detail. Future research should also explore the role of other confounding factors. For example, the influence of psychosocial and social-economic factors on the association between diabetes and depression needs to be elucidated.

In conclusion, the present research adds to the evidence concerning the association between diabetes mellitus type II and depression, in that this association also holds in a longitudinal setting in a large general practice population. The present results indicate, however, that patients with diabetes mellitus are less likely to develop a subsequent depression than was expected based on previous research.

Acknowledgments The authors gratefully acknowledge the contributions of $\mathrm{N}$. Rozendaal in matching the patient data. We also like to express our gratitude towards all participating GP's in the RNH network and their patients. Without their continuous and careful registration, this work would not have been possible.

Duality of interest For none of the authors are there any dualities of interest to declare.

Open Access This article is distributed under the terms of the Creative Commons Attribution Noncommercial License which permits any noncommercial use, distribution, and reproduction in any medium, provided the original author(s) and source are credited. 


\section{Appendix}

Table 2 ICPC codes which are included as a confounding variable (number of co-morbid diseases)

\begin{tabular}{|c|c|}
\hline Disease description & ICPC code \\
\hline All malignancies & $\begin{array}{l}\text { A79, B72, B73, F74, D74, D75, D76, D77, N74, R84, R85, S77, T71, U75, } \\
\text { U76, U77, W72, X75, X76, X77, Y77, Y78 }\end{array}$ \\
\hline $\begin{array}{l}\text { Chronic gastro-intestinal } \\
\text { diseases }\end{array}$ & D92, D93, D94, D97 \\
\hline Peptic ulcera & D85, D86 \\
\hline Ischemic diseases & $\mathrm{K} 74, \mathrm{~K} 75, \mathrm{~K} 76$ \\
\hline $\begin{array}{l}\text { Pulmonary embolism and } \\
\text { phlebitis }\end{array}$ & K93, K94 \\
\hline Cerebrovascular diseases & K89, K90, K91 \\
\hline Arrhythmias & K78, K79, K80 \\
\hline Benign prostatic hypertrophy & Y85 \\
\hline Chronic cardiovascular & K74, K75, K76, K77, K78, K79, K80, K82, K83, K87, K89, K90, K91, K92 \\
\hline Heart failure & K77, K82 \\
\hline Mental disorders & $\mathrm{P} 28, \mathrm{P} 70, \mathrm{P} 71, \mathrm{P} 72, \mathrm{P} 73, \mathrm{P} 74, \mathrm{P} 75, \mathrm{P} 77, \mathrm{P} 79, \mathrm{P} 80, \mathrm{P} 85, \mathrm{P} 98$ \\
\hline Movement disorders & L84, L85, L88, L89, L90, L91, L95, L98, \\
\hline $\begin{array}{l}\text { Asthma, CPOD and } \\
\text { bronchitis }\end{array}$ & R91, R95, R96, \\
\hline Chronic respiratory diseases & R70, R75, R91, R95, R96, R97 \\
\hline Hormonal diseases & T85, Т86, Т90, Т92, Т93, Т99 \\
\hline $\begin{array}{l}\text { Eczema, psoriasis and } \\
\text { chronic ulcer skin }\end{array}$ & S87, S91, S97 \\
\hline Diseases of the eye & F83, F84, F94 \\
\hline Diseases of the ear & H83, H86, \\
\hline Diseases of the urinary tract & U04, U85, U88 \\
\hline $\begin{array}{l}\text { Diseases of the nervous } \\
\text { system }\end{array}$ & N89, N90, N92, N70, N85, N86, N87, N88 \\
\hline
\end{tabular}

\section{References}

1. Penninx BW, Beekman AT, Ormel J, Kriegsman DM, Boeke AJ, van Eijk JT, et al. Psychological status among elderly people with chronic diseases: does type of disease play a part? J Psychosom Res. 1996;40(5):521-34.

2. Barnard KD, Skinner TC, Peveler R. The prevalence of comorbid depression in adults with type 1 diabetes: systematic literature review. Diabet Med. 2006;23(4):445-8.

3. Li C, Ford ES, Strine TW, Mokdad AH. Prevalence of depression among US adults with diabetes: findings from the 2006 behavioral risk factor surveillance system. Diabetes Care. 2008;31(1): $105-7$.

4. Ludman EJ, Katon W, Russo J, Von Korff M, Simon G, Ciechanowski $\mathrm{P}$, et al. Depression and diabetes symptom burden. Gen Hosp Psychiatry. 2004;26(6):430-6.

5. Anderson RJ, Clouse RE, Freedland KE, Lustman PJ. The prevalence of comorbid depression in adults with diabetes. A meta-analysis. Diabetes Care. 2001;24(6):1069-78.

6. Gonzalez JS, Safren SA, Cagliero E, Wexler DJ, Delahanty L, Wittenberg E, et al. Depression, self-care, and medication adherence in type 2 diabetes: relationships across the full range of symptom severity. Diabetes Care. 2007;30(9):2222-7.
7. Lustman PJ, Clouse RE. Depression in diabetic patients: the relationship between mood and glycemic control. J Diabet Complications. 2005;19(2):113-22.

8. Brenes GA. Anxiety, depression, and quality of life in primary care patients. J Clin Psychiatry. 2007;9(6):437-43.

9. Kessing LV, Nilsson FM, Siersma V, Andersen PK. No increased risk of developing depression in diabetes compared to other chronic illness. Diabetes Res Clin Pract. 2003;62(2):113-21.

10. van den Akker M, Schuurman AG, Metsemakers JFM, Buntinx F. Is depression related to subsequent diabetes mellitus? Acta Psychiatr Scand. 2004;110:178-83.

11. Carnethon MR, Kinder LS, Fair JM, Stafford RS, Fortman SP. Symptoms of depression as a risk factor for incident diabetes: findings from the national health and nutrition examination epidemiologic follow-up study, 1971-1992. Am J Epidemiol. 2003; 158:416-23.

12. Knol MJ, Twisk JW, Beekman AT, Heine RJ, Snoek FJ, Pouwer F. Depression as a risk factor for the onset of type 2 diabetes mellitus A meta-analysis. Diabetologia. 2006;49(5):837-45.

13. Golden SH, Lazo M, Carnethon M, Bertoni AG, Schreiner PJ, Roux AV, et al. Examining a bidirectional association between depressive symptoms and diabetes. Jama. 2008;299(23): 2751-9. 
14. Kessing LV, Nilsson FM, Siersma V, Andersen PK. Increased risk of developing diabetes in depressive and bipolar disorders? J Psychiatr Res. 2004;38(4):395-402.

15. de Jonge P, Roy JF, Saz P, Marcos G, Lobo A. Prevalent and incident depression in community-dwelling elderly persons with diabetes mellitus: results from the ZARADEMP project. Diabetologia. 2006;49(11):2627-33.

16. Maraldi C, Volpato S, Penninx BW, Yaffe K, Simonsick EM, Strotmeyer ES, et al. Diabetes mellitus, glycemic control, and incident depressive symptoms among 70- to 79-year-old persons: the health, aging, and body composition study. Arch Intern Med. 2007;167(11):1137-44.

17. de Groot M, Doyle T, Hockman E, Wheeler C, Pinkerman B, Shubrook J, et al. Depression among type 2 diabetes rural appalachian clinic attendees. Diabetes Care. 2007;30(6):1602-4.

18. Buntinx F, De Lepeleire J, Heyrman J, Fischler B, Vander Mijnsbrugge D, Van den Akker M. Diagnosing depression: what's in a name? Eur J Gen Pract. 2004;10(4):162-5.

19. Metsemakers JF, Knottnerus JA, van Schendel GJ, Kocken RJ, Limonard CB. Unlocking patients' records in general practice for research, medical education and quality assurance: the registration network family practices. Int J Biomed Comput. 1996;42(12):43-50.
20. Metsemakers JFM, Höppener P, Knottnerus J, Limonard C. Computerized health information in the Netherlands: a registration network of family practices. Br J Gen Pract. 1992;42(356): $102-6$.

21. WONCA Cco. ICPC international classification of primary care. Oxford: Oxford Univesity Press; 1987.

22. Petrak F, Hardt J, Wittchen HU, Kulzer B, Hirsch A, Hentzelt F, et al. Prevalence of psychiatric disorders in an onset cohort of adults with type 1 diabetes. Diabetes Metab Res Rev. 2003; 19(3):216-22.

23. Baan CA JHH, Rijken PM. Afstemming in de zorg. Een achtergrondstudie naar de zorg voor mensen met een chronische aandoening. RIVM rapport. Bilthoven: RIVM/NIVEL; 2003.

24. Crombie DL, Cross KW, Fleming DM. The problem of diagnostic variability in general practice. J Epidemiol Community Health. 1992;46(4):447-54.

25. Kawakami N, Takatsuka N, Shimizu H, Ishibashi H. Depressive symptoms and occurrence of type 2 diabetes among Japanese men. Diabetes Care. 1999;22(7):1071-6.

26. van den Akker M, Buntinx F, Metsemakers JF, Roos S, Knottnerus JA. Multimorbidity in general practice: prevalence, incidence, and determinants of co-occurring chronic and recurrent diseases. J Clin Epidemiol. 1998;51(5):367-75. 